\title{
La clôture en question dans les internats de rééducation pour filles (1945-1975)
}

\section{Anne Thomazeau}

\section{OpenEdition}

1 Journals

\section{Édition électronique}

URL : https://journals.openedition.org/clio/6292

DOI : $10.4000 /$ clio.6292

ISSN : 1777-5299

Éditeur

Belin

Édition imprimée

Date de publication : 1 novembre 2007

ISBN : 978-2-85816-940-5

ISSN : $1252-7017$

\section{Référence électronique}

Anne Thomazeau, «La clôture en question dans les internats de rééducation pour filles (1945-1975) », Clio. Femmes, Genre, Histoire [En ligne], 26 | 2007, mis en ligne le 20 avril 2008, consulté le 23 avril 2022. URL : http://journals.openedition.org/clio/6292 ; DOI : https://doi.org/10.4000/clio.6292

Ce document a été généré automatiquement le 23 avril 2022

Tous droits réservés 


\title{
La clôture en question dans les internats de rééducation pour filles (1945-1975)
}

\author{
Anne Thomazeau
}

1 Les années 1940 à 1960 constituent une période décisive pour le renouvellement des méthodes de prise en charge des mineur-e-s désigné-e-s comme délinquant-e-s ou inadapté-e-s et placé-e-s en institutions spécialisées. En affirmant le primat de l'éducation sur la répression, l'ordonnance de février 1945 sur l'enfance délinquante illustre les évolutions alors en cours dans les maisons de correction, de redressement ou de relèvement. La volonté est forte de rompre avec le passé pénitentiaire et l'héritage carcéral, les éducateurs remplacent les surveillants, les anciennes structures sont réaménagées, de nouvelles sont créées. Ainsi, la direction de l'Éducation surveillée ouvre, en 1947, un nouvel établissement pour filles, l'institution publique de Brécourt, présentée comme un modèle. Dans le même esprit, les congrégations religieuses comme celle du Bon Pasteur d'Angers ${ }^{1}$ - qui, jusqu'à la fin des années 1960 gèrent la grande majorité des établissements pour filles ${ }^{2}$, réorganisent leurs établissements et réforment leurs méthodes. Le mouvement de réforme touche donc l'ensemble du secteur, comme l'indique la terminologie qui s'impose progressivement pour désigner ces institutions : internats ou maisons de rééducation.

2 Ouvrir les établissements sur le monde dit extérieur est un des principes de la rééducation. Cependant, cette volonté d'ouverture rencontre plusieurs obstacles. D'abord, les structures héritées d'avant-guerre sont le plus souvent caractérisées par leur fermeture ${ }^{3}$ : les religieuses étant elles-mêmes cloîtrées, les mineures " partage[nt] en somme [leur] clôture » ${ }^{4}$ et l'établissement public de Cadillac a, en 1944, un « aspect de prison ${ }^{5}$. Ensuite, la persistance d'impératifs contradictoires tels que la coupure avec le milieu et la protection contre les mauvaises tentations freinent l'ouverture, en particulier dans le cas des filles.

3 Étudier les différentes brèches successivement creusées dans la clôture initiale met en lumière certaines des spécificités de la rééducation féminine. Ainsi, essayer de cerner 
pour quelles raisons les acteurs, tout en critiquant de plus en plus explicitement la clôture des établissements, ne la remettent pas totalement en cause permet de saisir les tensions existantes entre la volonté de réforme et la persistance d'une lecture traditionnelle, en termes moraux et sexuels, de la déviance juvénile féminine. Toutefois, à mesure que le regard porté sur les mineures et sur leur famille, et donc sur leur prise en charge, évolue, une réelle ouverture est progressivement réalisée, selon deux modalités principales: faire entrer des éléments extérieurs et autoriser les mineures à sortir.

\section{« Le paradoxe de l'internat ${ }^{6}$}

4 «Les Instituts de réforme n'ont pas pour but d'enfermer des jeunes filles débauchées pour en débarrasser la rue, mais de rendre à la société des femmes fortes et saines, capables de fonder une famille solide et prospère, de tenir convenablement leur foyer " 7. Cet extrait d'une enquête sur les institutions de filles en 1950, laisse apparaître le caractère paradoxal de la prise en charge: enfermer, donc couper du monde, pour mieux réadapter au monde. Dans cette logique, comme l'écrit Jean-Marie Fecteau à propos des établissements québecois du XIXe siècle, « l'enfermement est une pratique d'initiation au monde adressée à ceux qui sont censés être incapables de s'y adapter " ${ }^{8}$. Cette contradiction, au moins apparente, entre l'objectif et les méthodes de la prise en charge n'est pas nouvelle, puisqu'elle concerne également les établissements d'avantguerre et du XIXe siècle. Cependant, à partir des années 1940 et surtout 1950, la conscience de ce "paradoxe de l'internat qui veut que l'on mette en vase clos ceux à qui il faut apprendre la liberté " ${ }^{9}$ devient si vive qu'elle conduit les acteurs à s'interroger sur la pertinence et l'efficacité de cet enfermement pour préparer la liberté future. Comme le remarque une religieuse au tout début des années 1960 :

En raison de ce qu'il a de clos et d'artificiel d'une part, de soustrait à la vie sociale d'autre part, l'internat peut en réadaptant une jeune au point de vue fonctionnel ou psychique, la désadapter du point de vue social. [Il y a un] danger permanent d'échec le jour de la remise en liberté car l'adolescente devra nager sans avoir connu l'eau ${ }^{10}$.

5 Cependant, il ne s'agit pas, avant la fin des années 1960, de remettre en cause le principe même de l'internat. Ce type d'établissement reste considéré comme une « nécessité absolue », soit " par suite des carences du milieu » ${ }^{11}$, soit en raison de la personnalité du jeune. Il paraît alors indispensable, pour les filles comme pour les garçons, de marquer une rupture nette avec la vie passée afin de rééduquer la personnalité. Selon le juge des enfants Jean Chazal, le processus de rééducation compte trois étapes : le déconditionnement, le reconditionnement et la personnalisation grâce à un cadre permettant l'épanouissement ${ }^{12}$. Or, l'idée est alors largement partagée que, pour réaliser un tel objectif, il est préférable que l'enfant soit, au moins dans les premières étapes, exposé à une seule influence, celle de l'internat, et protégé des influences néfastes de son milieu.

De plus, dans le cas des filles, la nécessité d'une coupure avec l'extérieur est également liée à la façon dont est perçue la déviance féminine. Non seulement celle-ci paraît socialement moins bien tolérée que celle des garçons, mais elle est, le plus souvent, l'objet d'une lecture morale. «La délinquance féminine est pathologie, vice ou péché, avant d'être illégalisme; la femme délinquante est d'abord une "femme tombée ", moralement "déchue" ", avant d'être légalement coupable, constate Claudie Lesselier 
dans son étude sur les prisons de femmes entre 1820 et 1939, avant de résumer cela par la notion de "double transgression féminine ${ }^{13}$ ", transgression légale mais surtout transgression morale. Après la Seconde Guerre mondiale, un grand nombre de mineures sont placées en raison de leur inconduite. Ainsi Odette Philippon qualifie indistinctement les mineures placées de "débauchées " tandis que la directrice de Brécourt écrit : "sauf en de rares exceptions, le malheur de nos pupilles a son origine dans les relations sexuelles ${ }^{14}$. Par conséquent, restaurer la moralité des mineures, "les détourner de la prostitution; les préparer à leur rôle de future maitresse de maison, d'épouse, de mère ${ }^{15}$ sont parmi les principaux objectifs de la rééducation. De ce fait, rien ne paraît plus pertinent que d'éloigner ces jeunes filles des éventuelles tentations et des mauvaises fréquentations, en les plaçant dans un monde clos et presque exclusivement féminin. Les jeunes filles semblent d'ailleurs être plus fréquemment placées en internat que les garçons et l'ouverture des établissements féminins sur l'extérieur paraît moins rapide ${ }^{16}$. A titre d'exemple, en 1961, les filles de Brécourt ne bénéficient pas de sorties libres le dimanche contrairement aux garçons $\mathrm{du}$ même âge également placés en établissements publics ${ }^{17}$.

7 A partir des années 1950 surtout, se trouvent donc juxtaposées deux appréciations contradictoires sur la clôture considérée à la fois comme une nécessité et un problème : elle paraît indispensable pour marquer une coupure avec la vie passée et restaurer la moralité, mais elle est un obstacle à la préparation de la vie future. Pour tenter de résoudre cette contradiction, les acteurs tentent de compenser les effets de l'enfermement, notamment en mettant en place une organisation "aussi proche que possible de la vie réelle [dans laquelle] le rythme de vie des élèves est celui de jeunes filles normales vivant dans leur famille ${ }^{18}$. Concrètement, il s'agit de diviser l'effectif en petits groupes vivant chacun dans leur appartement autour de leur éducatrice, de séparer les lieux de vie dite familiale des lieux de formation scolaire et professionnelle et de suivre un emploi du temps rigoureux. Finalement, pour atténuer les effets de la clôture externe, l'espace interne est divisé par l'introduction de cloisonnements, ou en quelque sorte, de clôtures internes, aussi bien entre les groupes qu'entre les activités.

Parallèlement à ces aménagements, les acteurs insistent sur la nécessité de préparer la sortie des mineures, notamment en leur faisant acquérir une bonne formation professionnelle et ménagère. Cependant, la confrontation des mineures au monde extérieur apparaît rapidement comme la meilleure solution pour préparer leur sortie. Même si cette confrontation peut se faire lors d'une phase de transition grâce à un placement en foyer de semi-liberté, l'idée s'impose progressivement que la rééducation sera mieux réussie si ces contacts sont organisés avant la fin de la prise en charge, grâce à une ouverture progressive et maîtrisée des établissements. Cette évolution n'est pas sans lien avec les changements alors en cours dans la société française. D'une part, le système éducatif général est, pendant les Trente Glorieuses, en pleine mutation: explosion scolaire, diminution du nombre d'internes dans les établissements scolaires, " ouverture des lycées au monde extérieur ${ }^{19}$... D'autre part, la remise en cause de la clôture des religieuses est favorisée par le contexte général d'ouverture de l'Église catholique symbolisé par le Concile de Vatican II. 


\section{«Ouvrir les fenêtres » 20 : une ouverture indirecte et contrôlée}

9 La façon la plus simple, et en quelque sorte la moins risquée, d'ouvrir l'internat, est de laisser entrer des éléments du monde extérieur. Ces derniers peuvent être tout simplement des échos des problèmes sociaux et notamment les actualités. Comme l'écrit une éducatrice du Bon Pasteur d'Angers, "ouvrir un établissement sur l'extérieur ne consiste pas uniquement à envoyer les enfants « hors du centre ». Il y a tout un aspect de cette ouverture qui se fait dans l'internat. C'est là une question d'ambiance créée par les éducateurs dans la mesure où ils sont eux-mêmes ouverts aux problèmes de la vie extérieure. [...] Ce contact indirect avec la vie courante peut se faire aussi par l'intermédiaire de la radio, des journaux... » ${ }^{21}$. En pratique, l'écoute de la radio, et par la suite, de la télévision, même si elle est la plupart du temps contrôlée, prend une importance croissante dans les centres tandis que des cercles de discussion sur les problèmes d'actualité sont organisés.

10 Par ailleurs, dès les années 1940, certains établissements décident d'impliquer dans la rééducation des intervenants extérieurs, souvent membres du scoutisme. Ainsi, la Supérieure de Chevilly-Larue propose, dès 1946, à des cheftaines d'intervenir dans l'établissement pour en "ouvrir les fenêtres ». Leur rôle est clairement résumé lors d'une session sur les rapports du scoutisme et des établissements religieux de rééducation : elles sont « le reflet du monde extérieur » et « un exemple de ce que peut être une vraie jeune fille, par leur tenue et leurs propos " ${ }^{22}$. Les intervenant-e-s sont donc choisi-e-s avec soin et les visites des anciennes élèves ne sont pas toujours autorisées. De même, lorsque les établissements religieux sont conduits, notamment du fait du tarissement des vocations, à recruter du personnel laïc, les nouvelles éducatrices sont sélectionnées avec soin. L'arrivée de ces laïques est d'ailleurs considérée comme un moyen d'ouverture sur l'extérieur, puisque, par définition, les laïques n'appartiennent pas à la communauté.

11 Progressivement les institutions laissent de plus en plus entrer des personnes extérieures qu'elles n'ont pas choisies : les familles des mineures. Même si une certaine réticence demeure, l'idée se développe, à partir des années 1950, qu'» accepter un enfant, c'est l'admettre dans sa constellation familiale et, selon cette optique, la relation familiale cesse d'être un obstacle, mais est au contraire un moteur " ${ }^{23}$. Pour autant, les établissements ne s'ouvrent aux parents que progressivement: la correspondance est lue et éventuellement censurée, les parents doivent obtenir une autorisation de visite auprès du juge, les horaires et la fréquence des visites sont réglementées... Au début de la période, dans certains établissements au moins, les mineures, comme les religieuses, reçoivent les visites derrière une grille. Par la suite, la grille disparait, mais, jusque dans les années 1960 environ, les visites des proches se font presque toujours dans un lieu spécifique, encore souvent appelé parloir, et sous le regard d'un membre du personnel. Finalement, puisque les parents ne rencontrent pas leurs enfants dans leurs lieux de vie, ils ne pénètrent pas réellement dans la clôture avant les années 1960, voire les années 1970, selon les établissements.

En parallèle de cet effort d'ouverture de l'internat aux éléments extérieurs, un certain nombre de clôtures internes s'atténuent, et en particulier celles qui séparent la communauté des religieuses des autres habitants de l'internat et notamment le personnel laïc et les élèves ${ }^{24}$. Ainsi, la volonté de mettre en place une «authentique 
collaboration " ${ }^{25}$ entre les éducatrices religieuses et laïques atténue progressivement la clôture spatiale mais aussi symbolique que certaines laïques ont ressentie à leurs débuts. Par ailleurs, si une distance nette entre la communauté et les élèves a pu être considérée, par le passé, comme " thérapeutique, voire éthique entre le normal et l'anormal » ${ }^{26}$, cet écart diminue progressivement. Ainsi une religieuse du Bon Pasteur raconte que, dans les années 1950, les sœurs ne mangeaient pas avec les jeunes : « on était à table pour les servir [...] mais après on allait manger à la communauté ", puis elle précise que cette pratique "anachronique " ${ }^{27}$ a rapidement disparu. L'espace aussi est décloisonné : au Bon Pasteur de Bourges par exemple, le "grand parc qui était jusqu'alors réservé aux religieuses ${ }^{28}$ est ouvert aux élèves en 1965. Enfin, à partir de la fin des années 1960, les religieuses quittent progressivement leur habit.

Parallèlement à cette atténuation des clôtures externes et internes, de nouveaux cloisonnements se mettent en place pour délimiter un espace propre à chaque fille. Dans l'établissement public de Brécourt, l'effectif est non seulement divisé en petits groupes, mais des travaux sont réalisés pour que chacune possède une chambre individuelle. Lorsque les locaux anciens ne permettent pas ce type d'aménagement, les dortoirs sont divisés en boxes individuels par la pause de panneaux de bois. Ces modifications sont, bien sûr, le reflet d'une volonté d'individualiser la prise en charge et de laisser à chacune un espace personnel. Ils ont aussi pour effet de séparer les filles les unes des autres, ce qui est supposé permettre de mieux éviter des rapprochements interdits et redoutés : les amitiés dites douteuses et les relations homosexuelles.

En développant les contacts « centripètes » ${ }^{29}$ avec l'extérieur et en atténuant les clôtures internes, l'internat s'ouvre progressivement sur le monde. Cependant, cette ouverture est limitée puisque la jeune fille est en permanence sous le regard et le contrôle de l'institution.

\section{«Apprendre la liberté » 30 : sortir sans accompagnement}

Dès les années 1940, des sorties de groupe dominicales et des séjours hors de l'enceinte de l'établissement sont organisés. Ces sorties collectives, si elles sont l'occasion de contacts avec l'extérieur, restent caractérisées par la surveillance de l'institution et par une sorte d'entre soi que les mineures ne sont pas autorisées à quitter. Ainsi, une religieuse du Bon Pasteur écrit en 1978, que dans les années 1950, les sorties dominicales se faisaient « en rangs, comme si par cette mesure nous voulions repousser les gens qui auraient essayé de leur parler ${ }^{31}$.

C'est donc seulement en autorisant les élèves à sortir sans accompagnement que l'institution offre aux mineures la possibilité de réellement préparer leur sortie. Cependant, les fugues demeurent longtemps la principale façon d'avoir des relations avec l'extérieur hors du contrôle de l'institution. Elles sont facilitées par une des conséquences de la volonté d'ouverture: une diminution de la matérialisation de la clôture. A Brécourt, par exemple, les portes de l'établissement sont ouvertes notamment parce que, selon la directrice, " pour certaines délinquantes des impératifs moraux sont plus difficiles à supporter que de hautes murailles " ${ }^{32}$. Même si cela n'est pas autorisé, sortir seule n'est donc pas très difficile. Ainsi plus de la moitié $(55,6 \%)$ des 90 élèves de cet établissement étudiées ont fait une ou plusieurs fugue(s) ${ }^{33}$. Cependant, 
ces fugues sont redoutées, notamment par crainte de conséquences estimées très probables de la part de filles dont la conduite est jugée peu sérieuse: maladies vénériennes ou grossesse. De ce fait, les fugues sont sévèrement sanctionnées dans tous les établissements, parfois par le port d'une tenue de punie ou par la coupe des cheveux, souvent par un séjour en cellule d'isolement ${ }^{34}$. Cette dernière, qui constitue une sorte de clôture dans la clôture, reste utilisée après 1945. Ainsi sœur Dublanc, décrit, en 1967, celle du centre de Chevilly: «Les murs et le sol sont cimentés, une grande fenêtre, à hauteur d'homme, grillagée à l'extérieur, leur permet de voir ce qui se passe à l'extérieur. Aucun meuble. Un rectangle, dans lequel sont posées à même le sol une paillasse et des couvertures, est limité par une barre de ciment fixée au sol. Une porte en fer, surmontée d'une ouverture grillagée donne sur un couloir " ${ }^{35}$. Sœur Dublanc précise, en outre, que cette cellule est utilisée soit pour sanctionner, soit pour prévenir des incidents, en cas, par exemple, d'excitation, de tensions dans le groupe ou encore à la demande d'une mineure qui souhaite être seule.

Ce souci d'éviter les fugues et les sanctions prises laissent deviner combien les relations avec l'extérieur sans surveillance inquiètent. Néanmoins, les acteurs jugent ces relations progressivement indispensables pour réellement préparer la sortie et la réadaptation sociale. Dans cet objectif, les établissements accordent des permissions dans la famille, d'abord au compte-gouttes et seulement aux plus méritantes ou à celles dont la sortie est proche. Puis, ces séjours dans la famille se généralisent et se rallongent tandis que le terme de vacances remplace celui de permissions. Si cette pratique se développe, les inquiétudes ne disparaissent pas. La crainte demeure, en effet, que "les mauvaises habitudes reprennent le dessus, que la suspension de l'emprise de l'institution ouvre la porte au dévoiement " ${ }^{36}$ et que les vacances " démoli[ssent] en quelques jours ou quelques semaines les résultats déjà obtenus » ${ }^{37}$, du fait notamment de la faible valeur éducative de la famille et du manque de sens moral de ces jeunes filles. En outre, lors des permissions, l'institution ne peut contrôler les activités, les fréquentations ou encore les lectures. Ainsi, au début des années 1950, la directrice de l'établissement de Brécourt écrit à la mère d'une élève : «Je vous fais parvenir le livre que Renée a rapporté de sa permission. Nous ne pouvons pas tolérer que Renée ait à l'internat Les fleurs $d u$ Mal " ${ }^{38}$. Néanmoins, les vacances hors de l'institution apparaissent finalement indispensables et les établissements organisent pour celles qui ne peuvent aller dans leur famille des séjours en familles d'accueil ou dans des colonies extérieures.

18 À la fin des années 1960, les possibilités de sorties libres se multiplient progressivement. Là encore cela concerne d'abord celles dont la sortie est proche, pour lesquelles, faute de place en foyers de semi-liberté, une sorte de période de transition est ménagée au sein même de l'établissement. Puis, peu à peu, ces possibilités de sortie sont généralisées à l'ensemble des élèves. Ainsi, à Brécourt, les élèves qui apprennent la coiffure, par exemple, font des stages de formation à l'extérieur ou bien encore travaillent dans des salons le samedi. Finalement, dans les années 1970, les élèves sont de plus en plus souvent autorisées à retourner régulièrement dans leur famille le weekend et à se promener sans accompagnement. Une des conséquences de cette ouverture croissante est la modification du regard porté par les acteurs sur la question de la sexualité des élèves : tout en cessant peu à peu d'être au cœur des préoccupations, elle devient moins taboue et il apparaît de plus en plus nécessaire de compléter l'éducation morale par une réelle éducation sexuelle ${ }^{39}$. 
L'internat des années 1970 ressemble finalement assez peu à celui des années 1940, en particulier en ce qui concerne l'ouverture. Comme le souligne le psychiatre du centre de Chevilly, en 1976, "nombre d'internats sont en fait parvenus d'ouverture en ouverture, à réaliser des formules éducatives qui rappellent étrangement celles du classique foyer de semi-liberté $"{ }^{40}$. Pour autant il n'est pas certain que l'internat a alors atteint « le terme ultime de [son] évolution » ainsi décrit par un éducateur en 1959 : «il ne sera plus à opposer au monde extérieur, [...] son nom même ne se justifiera plus et, quelle que soit l'aide dont un jeune pourra avoir besoin, il sera et restera dans le " monde ", qui n'aura plus à être qualifié d'» extérieur » " ${ }^{41}$. Effectivement, l'ouverture croissante n'implique pas une évolution proportionnelle de ce que les élèves ont ressenti. Au contraire, le champ lexical de l'enfermement reste souvent utilisé par les mineures pour parler de leur placement, comme le souligne une religieuse du Bon Pasteur en 1978: " la coupure, la clôture, autrefois très matérialisées, sont en voie de disparition, mais [...] elles se savent en internat donc toujours « enfermées » selon leur expression... ${ }^{42}$.

\section{BIBLIOGRAPHIE}

BANTIGNY Ludivine, 2005, « De la modernité dans le lycée des années 1950, l'adaptation de la culture lycéenne au « monde moderne » », in Pierre Caspard, Jean-Noël Luc et Philippe Savoie (dir.), Lycées, lycéens, lycéennes, deux siècles d'histoire, Paris, INRP, p. 269-281.

BOURQUIN Jacques, 1969, « Étude sur 55 adolescentes incarcérées au quartier des mineures de la prison de Fresnes », Rééducation, mars-avril-mai, p. 1- 60.

Collectif d'acteurs-témoins, 1991, De l'impossible à la chaîne d'amitié, 1945-1965, Paris, Équipes d'amitié.

DUMAS Claire et TÉTARD Françoise, à paraître, Les demoiselles de Bourges, Du Bon Pasteur à l'Éducation surveillée.

FECTEAU Jean-Marie, 2005, « L'univers religieux, l'éthique libérale et l'économie de l'enfermement. Le cas du Québec au XIXe siècle », in Bernard Delpal et Olivier Faure (dir.), Religion et enfermements (XVIIe-XXe siècles), Rennes, PUR, p. 225-238.

GIRAUD Sœur, 1999, « Le monde féminin du Bon Pasteur ", in Conservatoire national des archives et de l'histoire de l'éducation spécialisée, Elles ont épousé l'éducation spécialisée, Educatrices et femmes d'éducateurs il y a cinquante ans, Paris, L'Harmattan, p. 113-123.

KOEPPEL Béatrice, 1982, De la pénitence à la sexologie, Essai sur le discours tenu aux jeunes filles, Paris, Le Sycomore, Centre de formation et de recherche de l'Éducation surveillée.

LESSELIER Claudie, 1984, « Les femmes et la prison, 1820-1939 », in Jacques-Guy Petit (dir.), La Prison, le bagne et l'histoire, Paris, Les Méridiens, 1984, p. 115-128. 
PECRIAUX Christiane, 2005, Le Couvent à sabots, témoignage autobiographique, Nantes, Editions Amalthée.

PROST Antoine, 2004 (1ère éd. 1981), L'École et la Famille dans une société en mutation (depuis 1930), tome IV de l'Histoire de l'enseignement et de l'éducation, Paris, Perrin.

QUINCY-LEFEBVRE Pascale, 1997, Familles, institutions et déviances, Histoire de l'enfance difficile (1880fin des années 1930), Paris, Économica.

RIEHL D., « La réforme de l'Institution publique d'Education surveillée de Cadillac », Revue de l'éducation surveillée, mai-juin 1946, n², p. 59.

VILBROD Alain, 2005, «Grandir tant bien que mal derrière les hauts murs d'une institution religieuse : les orphelins-apprentis d'Auteuil », in Bernard Delpal et Olivier Faure (dir.), Religion et enfermements (XVIIe-XXe siècles), Rennes, PUR, p. 55-73.

\section{NOTES}

1. L'expression "Bon Pasteur» a été utilisée pour désigner toute maison de rééducation religieuse, mais toutes n'étaient pas gérées par la congrégation du Bon Pasteur d'Angers.

2. On dénombre environ 100 à 120 internats pour filles dans les années 1960 . Moins de 5\% sont publics, presque les trois quarts sont gérés par des congrégations religieuses et le reste par des associations laïques.

3. Quincy-Lefebvre $1997: 222-224$.

4. Giraud 1999 : 115. La clôture des religieuses n'implique pas une fermeture totale puisqu'elles peuvent sortir pour l'œuvre de la congrégation, notamment pour accompagner les filles.

5. Riehl 1946.

6. Sr Marie-Thérèse, Les loisirs, moyen d'ouverture de l'internat sur le monde extérieur, mémoire pour le diplôme d'éducatrice, 1963, p. 62, Archives du Bon Pasteur d'Angers (BPA).

7. O. Philippon, La jeunesse coupable vous accuse, les causes familiales et sociales de la délinquance juvénile, Paris, Sirey, 1950, p. 170.

8. Fecteau 2005 : 232.

9. Sr Marie Thérèse, Les loisirs..., op. cit., p. 62.

10. Sr Marie de Sainte Françoise, Vacances en famille pour les élèves des internats de rééducation, mémoire pour le diplôme d'éducatrice, début des années 1960, p. 17-18, BPA.

11. Dr Bascou, «Les relations familiales », Sauvegarde de l'enfance, janv-mars 1960, n 1-3, p. 67.

12. J. Chazal, L'enfance délinquante, Paris, PUF, Que sais-je ?, 1953, p. 74.

13. Lesselier $1984: 116$.

14. D. Riehl, "L'éducatrice d'IPES ", compte-rendu, première session de formation des éducateurs, 1947, archives de Savigny-sur-Orge, sans cote.

15. D. Riehl, « L'éducatrice d'IPES »...

16. Bourquin $1969: 4$.

17. « Le problème des sorties du dimanche dans les internats publics d'éducation surveillée », sd., CAC, 91 0300-48.

18. D. Riehl, «La rééducation des filles, l'expérience de Brécourt », Revue pénitentiaire et de droit pénal, juil-sept 1956, n 7-9, p. 742.

19. Bantigny $2005: 269$. Voir aussi Prost 2004.

20. Cité dans Collectif d'acteurs-témoins $1991: 27$.

21. J. Dordonnat, Ouverture sur le monde extérieur des internats pour inadaptés, mémoire pour le diplôme d'éducatrice, 1960, p. 104, BPA. 
22. "Session sur les rapports du scoutisme et des établissements religieux de rééducation en vue de la réadaptation sociale », 1949, p. 43 et 32, Archives de Chevilly-Larue.

23. Dr Bascou, « Les relations familiales »..., p. 66.

24. Il faut aussi évoquer les Sœurs Madeleine, élèves devenues religieuses contemplatives, qui vivent entre elles, dans une sorte de double clôture, et les Sœurs auxiliaires, élèves restées dans l'établissement pour s'occuper des mineures.

25. Sr Marie-Pierre, Collaboration des éducatrices religieuses et lä̈ques dans les établissements à structure religieuse, mémoire pour le diplôme d'éducatrice, 1967, p. 49, BPA.

26. Fecteau 2005 : 230.

27. Entretien avec Sœur M., religieuse du Bon Pasteur d'Angers, février 2004.

28. Dumas et Tétard, à paraître.

29. Sr Marie Thérèse, Les loisirs..., p. 42.

30. Sr Marie-Thérèse, Les loisirs..., p. 62.

31. R. Arcellin, Réflexion sur l'internat, hier, aujourd'hui, demain, 1978, p. 8, BPA.

32. D. Riehl, « La rééducation des filles »..., p. 741. L'établissement de Brécourt, dans le Val-d'Oise, était isolé et mal desservi ce qui rendait l'ouverture des portes plus facile qu'en centre ville.

33. Dossiers des mineures, Brécourt, $688 \mathrm{~W} 1$ à 526, AD Val-d'Oise.

34. Voir, par exemple le témoignage de Pecriaux 2005.

35. Sr Dublanc, «L'isolement, rôle préventif et curatif de l'isolement en centre d'observation pour adolescentes inadaptées ", Cahiers psycho-pédagogiques, n 54, juillet 1967, p. 13-14.

36. Vilbrod : 61 .

37. P. Meignant, «Les vacances », Sauvegarde de l'enfance, janv-mars 1960, n 1-3, p. 185.

38. Dossier T 34, Brécourt, AD Val-d'Oise, 688 W 495.

39. Koeppel 1982.

40. P. Le Moal, «Feu les « internats de rééducation » ", Sauvegarde de l'enfance, janv-fév 1976, n $1-2$, p. 48

41. J. Guyomarc'h, «Les relations à l'occasion des sorties provisoires ou définitives ", Sauvegarde de l'enfance, janv-mars 1960, n 1-3, p. 154.

42. R. Arcellin, Réflexion..., p. 17.

\section{RÉSUMÉS}

La clôture en question dans les internats de rééducation pour filles (1945-1975). À la Libération, la plupart des établissements qui prennent en charge les mineures placées par l'autorité judiciaire en raison de leur délinquance ou de leur inadaptation sont des institutions fermées et la grande majorité d'entre elles est gérée par des religieuses soumises, par leurs voeux, à la clôture. Cependant, les années d'après-guerre constituent une période décisive pour le renouvellement des méthodes de l'Education surveillée : l'accent est mis sur la réinsertion sociale et donc sur la nécessaire ouverture des établissements. Comment se traduit concrètement cette volonté d'ouverture dans les institutions féminines? Analyser les différentes modalités de l'ouverture faire entrer des éléments extérieurs dans l'internat d'une part, et d'autre part autoriser les mineures à sortir -, apporte des éclairages sur les représentations des acteurs concernant la déviance juvénile féminine et permet de saisir certaines des spécificités de la rééducation féminine 
Confinement called into question in reform schools for girls (1945's-1975's). In 1945 in France most reform schools for girls were closed institutions, and the majority were run by nuns subject themselves to religious enclosure. The post-war period witnessed a decisive change in reform school methods as the goal of social reintegration encouraged the opening of institutions. How, concretely, did this commitment to opening function within feminine institutions? An examination of how outside elements were admitted inside the reform school and the permissions for the minors to go out sheds light on contemporary representations of feminine juvenile delinquance and on certain specificities of feminine reeducation.

\section{INDEX}

Keywords : delinquance, boarding schools, correctional education, inadaptation, female juvenile delinquance, religious orders, reeducation

Mots-clés : délinquance, congrégations religieuses, éducation surveillée, inadaptation, internat, jeunes filles délinquantes, rééducation

\section{AUTEUR}

\section{ANNE THOMAZEAU}

Anne THOMAZEAU, professeur agrégée d'histoire-géographie, prépare un doctorat d'histoire contemporaine sur la rééducation en internat des jeunes filles délinquantes et inadaptées, de la Libération aux années 1970, à l'ENS-LSH, sous la direction d'Anne-Marie Sohn et de Jean-Noël Luc. 